793 THE "ON-CALL" SCHEDULE AND PERFORMANCE IN THE INTRAINING EXAM OF THE AMERICAN BOARD OF PEDIATRICS. Joseph R. Tobin and Frank A. Oski. SuNy, Upstate
Dept. Of Pediatrics, Syracuse, New York 13210. Medical Center, Dept. Of Pediatrics, Syracuse, New York pediatrics is a valuable means of monitoring acquisition of pediatrics is a valuable means of monitoring acquisition of the course of residency training. We examined the consequences of being "on-call" the night before the resident takes the exam using data collected retrospectively during the past 5 years.

The wilcoxon Sign Rank Test was used for house officers who had taken the exam in consecutive years under both conditions (on-call once; not on-call). The results of the Sign Rank Test demonstrated a significant detrimental effect $(p=0.05)$ for being on-call the night prior to the examination, although the mean scores under the two conditions were not strikingly difberent. The mean score for the residents who were on-call was 78.8 percentile; when the same residents took the exam after a night not on-call they achieved a mean score of 82.5 percentile. The impact of being on-call for bemale residents $(-6.5)$ was greater than for male residents $(-2.4)$. Great individual variation was observed with comparative scores ranging from -35 to +17 percentile when the resident took the exam after being on-call.

It is concluded that an individual's exam performance can be significantly altered by being "on-call" the night before the exam and this factor should be taken into account when the scores are interpreted.

794 VARICELLA-ZOSTER INFECTIONS IN PEDIATRIC RESIDENTS: A GROWING CONCERN. Armisa C. Tongson, Yvonne Lue, Johanna Goldfarb, Aditya Kaul and Donald S. Gromisch. (Sponsored by Lawrence R. Shapiro). New York Medicál College, Department of Pediatrics, Valhalla, New York.

Acute Varicella-Zoster (V.Z.) infections in adults are rela tively uncommon in the developed world. During the last 4 years 11 pediatric residents developed chickenpox. Of these, 7 were in the first year of training (PL1), 2 were PL2 and 1 PL3. Of the
the the first year of training (PL1), 2 were PL2 and 1 PL3. Of the
7 PL1 residents, 6 acquired infection within 6 months of begin7 pLl residents, 6 acquired infection within 6 months of beginable. All these young adults were born outside the U.S., 3 in the Philippines, 3 in India, 4 in Lat in America and 1 in Africa. Nine of the residents acquired V.Z. from casual contact with patients. All residents recovered without any untoward systemic complications. The only morbldity was extensive cutaneous involvement with some residual scarring. Duration of fever ranged from 2 to 6 days whereas time lost from work ranged from 7 to 20 days. Of great concern was occurrence of infection during the days. Of great concern was occurrence of infection during the
second trimester of pregnancy in 2 residents, one of which resecond trimester of pregnancy in 2 residents, one of which re-
sulted in stillibirth. Another resident developed her infection while working in the neonatal I.C.U. No secondary cases of V.z. were reported among patients cared for by these residents. Significant risk exists, however of nosocomial spread of V.Z. in high risk areas such as oncology or transplantation units.

Routine serologic screening for susceptibllity to V.Z. should be part of employment examination for pediatric residents, particularly in training programs with sizable numbers of foreign
born residents.

+795 THE ILL CHILD IN PAINTINGS. H.-R. Widemann, Christian-Albrechts-Universitat, Department of
Pediatrics, Kiel, Federal Republic of Germany.

To date, this subject has rarely been treated systematically. Here it is dedicated to the painting of western civilization beginning with the Italian Renaissance. During this time the child again began to play a greater role in western art, especially in Burgundian-Dutch and Italian painting. And since that time many artists have portrayed $i 11$, injured, or ma 1formed children.

The poster will show 25 to 30 paintings, beginning with depiction of an accident and three examples of the sick child sensu stricto, in some cases possibly permitting a diagnosis. Finaliy, there will be several examples of depictions of neurological or phychiatric problems.
796 DEVELOPMENTAL DELAY IN CHILDREN EXPOSED TO CHLORIDE 796 DEFICIENT FORMULA. Anne D. Willoughby, Howard A. Berendes. (Spon. by Sumner J. Yaffe). National Institutes of Health, National Institute of Child Health and Human Development Bethesda, Maryland.

During 1978-1979, more than 20,000 infants may have been exposed to chloride-deficient formula (CDF). A number of these children were noted to suffer from severe metabolic alterations, principally hypochloremic metabolic alkalosis (HMA). The present study examined 21 children who were exposed to CDF and developed HMA. Pediatric records were reviewed, medical and feeding histories were obtained from the parents, and children were administered the Bayley Scales of Infant Development (BSID). The range of age at testing was 13 to 29 months (mean=23.2). The Bayley Mental Development Index (MDI) ranged from 67 to 127 (mean=98.5) Length of exclusive use of CDF without supplementation with solids or other milk/formula (LENEXC) had a significant negative correlation with MDI $(r=-.55, p=.01)$. When length of exclusive use of CDF, age of onset of CDF use and age at testing were entered into a multiple regression analysis, LENEXC remained associated with MDI $\left(R^{2}=.39, p<.05\right)$. Sixteen of these children were re-examined at ages ranging from 40 to 51 months (mean=43.8) using the McCarthy Scales of Children's Abilities. There was a significant negative correlation between LENEXC and the perceptual-performance scale $(r=-.51, \mathrm{p} L .05)$ and between LENEXC and the motor scale $(r=-.52, p<.05)$. These data raise concern about the long-term outcome of these children.

797 NEONATAL STABILIZATION at COMMUNITY hospitals is IMPROVED BY OUTREACH EDUCATION: A PROSPECTIVE STUDY. John E. Wimmer, Jr., Nancy S. Edwards, Michael D. Cruze, Cathy Conklin, Patricia Heniford, Arthur E. Kopelman (Spon. by Jean Kenny). East Carolina University School of Medicine, Pitt County Memorial Hospital, Department Pediatrics, Greenville, NC.

We describe the first prospective evaluation of the effects of an outreach education program on pre-transport stabilization of sick neonates born in community hospitals. The relationship between the adequacy of stabilization and the timing of participation in a perinatal outreach program was studied. Information regarding stabilization procedures done by referring hospital personnel prior to the arrival of our transport team was recorded and expressed as the percentage of appropriate actions. Highly significant improvement was seen in these percentage scores in the six-month period folseen in these percentage scores in the six-month period fol-
lowing outreach education compared to the 12 months immediately preceding the educational program (mean post-outreach score $81 \%, n=58$ transports versus pre-outreach score $68 \%, n=40$, $\mathrm{p}<.001)$. In contrast, no spontaneous improvement with time (i.e. without outreach education) was seen during the two years prior to the program (mean pre-outreach score A $64 \%$, $\mathrm{n}=34$, pre-outreach score B $68 \%, \mathrm{n}=40$, non-significant).

This study shows that neonatal outreach education improves the stabilization of neonates in community hospitals and implies a beneficial effect on neonatal outcome.

\section{THE MICROFLORA OF ANIMALS USED IN HOSPITAL PET 798 THERAPY: IS THERE PATIENT RISK? Terry Yamauch} University of Arkansas for Medical Sciences aebbee Carter Children's Hospita1, Department of Pediatrics, Little Rock, Arkansas.

Pet therapy involves allowing children to visit their pets while in the hospital and/or the introduction of other animals into the hospital setting for viewing and entertaining the patients. Animals involved in pet therapy are numerous, but generally involved those that can be handled and transferred without much difficulty. Using standard microblological culture techniques, animals commonly used in pet therapy, school study, and pet loaning programs were analyzed for microblological flora. Cages, water and environmental materials were also cultured. Animals in this study included, salamanders, alligator, rat, tree frog, tarantula, milk snake salamanders, alligator, rat, tree frog, tarantula, milk snake,
turtle, owl and skink. Multiple microorganisms were recovered turtle, ow1 and skink. Multiple microorganisms were recovere
from all animals except the tarantula. Salmonella sp. was from all animals except the tarantula. Salmonella sp. was recovered from all reptiles at least once during the study
pertod. Serratia marcescens and Pseudomonas aeruginosa were also of ten recovered from an imals $\frac{\text { sendomonas }}{\text { and water }} \frac{\text { aeruginosa }}{\text { n cages. Severa }}$ animals were colonized with multiple gram-negative bacterfa. The outside of cages were of ten contaminated with gram-positive and gram-negative microorgan1sms. Although no outbreaks of Infection have been 1dentifled st our institution, unt11 further data is avallable, it seems logical to restrict the areas of the hospital into which these potential vehicles of infection can be utilized. 